\title{
Measurement based method for online characterization of generator dynamic behaviour in systems with renewable generation
}

\author{
Panagiotis N. Papadopoulos, Member, IEEE, Theofilos A. Papadopoulos, Senior Member, IEEE, \\ Andreas I. Chrysochos, Member, IEEE, Jovica V. Milanović, Fellow, IEEE
}

\begin{abstract}
This paper introduces a two-step methodology for online identification of the participation of generators in power system oscillatory modes, based on measured responses. The dominant modes in generator measured responses are initially identified using a mode identification technique and then introduced, in the next step, as input into a clustering algorithm. Critical groups of generators that exhibit poorly or negatively damped oscillations are identified, in order to enable corrective control actions and stabilize the system. The uncertainties associated with the operation of modern power systems with Renewable Energy Sources (RES) are investigated as well as the impact of the dynamic behavior of power electronic interfaced RES.
\end{abstract}

Index Terms - clustering, online dynamic security assessment, renewable generation, uncertainties, unsupervised machine learning.

\section{INTRODUCTION}

$\mathrm{D}$ URING the past years there has been a substantial increase in the installation of Renewable Energy Sources (RES), mainly driven by climate change and various social, economic and technical reasons. RES are intermittent in nature which is one of the main reasons for the change in power system operating conditions, power flows and topology. This coupled with the fact that they exhibit different dynamic behaviour than conventional generators leads to both spatial and temporal variation in power system dynamics and consequently operation under high uncertainty. Apart from the

This work was supported by the EPSRC project ACCEPT under EPSRCIndia collaboration scheme (grant number: EP/K036173/1).

The work of A. I. Chrysochos was conducted in the framework of the act "Support of Post-Doc Researchers" under the Operational Program "Human Resources Development, Education and Lifelong Learning 2014-2020", which is implemented by the State Scholarships Foundation and co-financed by the European Social Fund and the Hellenic Republic.

P. N. Papadopoulos and J. V. Milanović are with the School of Electrical and Electronic Engineering, The University of Manchester, Manchester, M60 1QD, U.K. (e-mail: milanovic@manchester.ac.uk, panagiotis.papadopoulos@manchester.ac.uk).

T. A. Papadopoulos is with the Power Systems Laboratory, Dept. of Electrical \& Computer Engineering, Democritus University of Thrace, Xanthi, Greece, GR 67100, (e-mail: thpapad@ee.duth.gr).

A. I. Chrysochos is with Cablel® Hellenic Cables S.A., Viohalco Group, GR 20100, Sousaki Korinthias, Korinthos, Greece, (e-mail: achrysochos@fulgor.vionet.gr). direct impact caused by RES, the displacement of synchronous generation and consequent inertia reduction is also affecting significantly the power system dynamic behaviour. These changes might lead to system operation closer to the stability limit and hence more prone to instability and ultimately blackouts.

In this context, close to real-time identification of the dynamic behavior of power systems and impeding instability that enables the application of corrective control actions becomes increasingly attractive. Moreover, the wide availability of measured data from Phasor Measurement Units (PMUs) coupled with the advances in data analytics and machine learning provide an array of tools that can be effectively used for such purposes.

Various data mining techniques, Decision Trees (DTs) [1][3], Ensemble Decision Trees (EDTs) [4], [5], Support Vector Machine (SVM) [6] and Artificial Neural Networks (ANNs) [7] have been used in online dynamic security assessment. Most commonly, the prediction focuses on binary classification, i.e. whether the system will remain transiently stable or not. There have been also some approaches [5], [8] for grouping of unstable generators after the fault is cleared. While this information is valuable for assisting corrective control actions, such as controlled islanding, these approaches focus mainly on aperiodic (first swing) instability. They do not provide any information regarding the oscillatory behavior of generators in case they are first swing stable (including potential oscillatory instability), which is within the scope of the proposed method in this paper.

The concept of slow coherency is about identifying generator groups that tend to swing together and can therefore represent oscillatory behavior of generators [9]. Measurementbased methods have also been developed to identify generator coherent groups [10], [11]. However, information from this type of methods focus on identifying coherent groups of generators that tend to swing together but do not directly provide information on the damping of oscillations after a specific disturbance, which is very important when further corrective control action needs to be taken. In [12], classification trees are used to predict well or poorly damped oscillations using power flow data, however, these trees need to be trained before their application in an online manner. In [13], a method for the online clustering of the oscillatory 
behaviour based on features extracted from Recurrence Quantification Analysis (RQA) is presented. The extracted features from RQA do not provide a direct physical meaning on the oscillatory behaviour of the generators though, especially when distinguishing between poorly and negatively damped oscillations. Furthermore, different methods based on the identification of parameters of oscillatory modes directly from time domain (TD) responses have been used in the past. They include Prony method [14], the Eigenvalue Realization Algorithm (ERA) [15] and the Matrix Pencil (MP) method [16]. Alternatively, there are also methods that derive the dominant modes using frequency domain (FD) responses [17] or a combination of the above as in the recently proposed hybrid FD/TD approach [18]. Such methods extract the dominant modes of measured responses with relatively high accuracy but do not provide any information on the participation of individual generators in these oscillatory modes.

This paper proposes, an integrated online method based on PMU measurements for the identification of generator oscillatory behavior in case of first swing stable response of the system. The proposed method enables the application of more effective corrective control by providing additional actionable information for system operators considering the participation of individual generators in poorly or negatively damped oscillatory modes following a disturbance. Additionally, it deals with practical application aspects when applied to a large number of cases for varying operating conditions imposed by RES intermittent behaviour. The distinct contributions of the proposed method are: 1) Mode identification is applied to TD responses of individual generators to identify both stable and unstable modes contrary to a single point of measurement usually used in the literature [14]-[18]. 2) Two post-processing techniques are incorporated, i.e. surplus mode filtering and sliding window, to deal with challenges introduced by using a fixed model order and slowly growing oscillatory modes, respectively. 3) The generators are clustered into groups in the second step of the method, based on the participation of individual generators in poorly or negatively damped oscillatory modes which is an additional feature compared to existing mode identification methods [14]-[18]. 4) Un-supervised machine learning is used to derive the generator groups without the need of generating simulated scenarios for training. 5) Monte Carlo (MC) simulations can be performed offline to investigate the probabilistic oscillatory behaviour of individual generators taking into consideration uncertainties of modern power systems (including RES).

\section{PROPOSED METHODOLOGY}

Once a contingency is identified as first swing stable, the proposed two-step method is applied to identify possible groups of generators that exhibit poorly or negatively damped oscillations, as shown in Fig. 1. Therefore, the proposed method can be seen as complementary to the method presented in [5] as it first addresses aperiodic instability and then the proposed method in this paper, oscillatory instability.

The first step of the proposed methodology involves the application of MP on the rotor angle responses of each generator obtained from available measuring infrastructure or PMU measurements. This is in order to identify the dominant modes contained in the oscillatory responses of each generator [19]. It should be noted that in this paper, simulations are used as a substitute for measurements to investigate the performance of the proposed method under a very large number of cases. Additionally, a surplus mode filtering approach is applied as part of the first step, to ensure consistency during the clustering procedure in the second step as well as to automate the procedure when dealing with a large number of case studies under different operating conditions.

The second step involves the application of a clustering method, namely the k-medoids algorithm [20], to cluster generators in relevant groups, i.e., exhibiting well or poorly/negatively damped modes using the least damped extracted modes identified in the previous step as clustering features. Therefore, the participation of individual generators in poorly or negatively damped modes can be derived in this manner. This is an added feature to measurement-based mode identification techniques and thus detailed information can be provided to system operators when designing and activating corrective control actions.

In addition, a sliding window approach can be applied, if needed, to capture slowly growing oscillations that might appear at a later time (outside of the selected initial time window).

Finally, by applying the proposed procedure to MC dynamic simulations performed offline, the probabilistic oscillatory behaviour of individual generators is investigated.

The proposed method offers a systematic approach regarding online identification of poorly/negatively damped oscillatory modes and the participation of individual generators in them as well as with practical aspects that arise from the application of mode identification techniques on a very large number of diverse cases for different operating conditions. It should also be noted that the different dynamic behaviour of power electronic interfaced units, including the effect of their uncertain behaviour causing a wider variation in operating conditions, as well as the consequent inertia reduction, are part of the motivation for the development of the proposed method. The aforementioned RES behaviour justifies the need for such online methods and is therefore considered in this paper as explained in the following Sections. The method is generalized in the sense that any mode identification and clustering method can be used as part of the proposed two-step procedure. The rest of this section describes each step in detail.

\section{A. Mode Identification}

The power system ringdown response $y(t)$ following a perturbation can be approximated by a sum of $N$ damped sinusoids (modes) [21].

$$
\hat{y}(t)=\sum_{i=1}^{N} A_{i} e^{\sigma_{i} t} \cos \left(\omega_{i} t+\varphi_{i}\right)
$$

where $\lambda_{i}=\sigma \pm \mathrm{j} \omega$ are the system eigenvalues and $\omega_{i}=2 \pi f_{i}, \sigma_{i}$, 
$A_{i}, \varphi_{i}$ are the angular frequency, damping, amplitude and phase angle of the $i$-th mode, respectively. The discrete-time representation of (1) can be written as (2), given that the record consists of $N_{w}$ equally $\Delta t$-spaced samples.

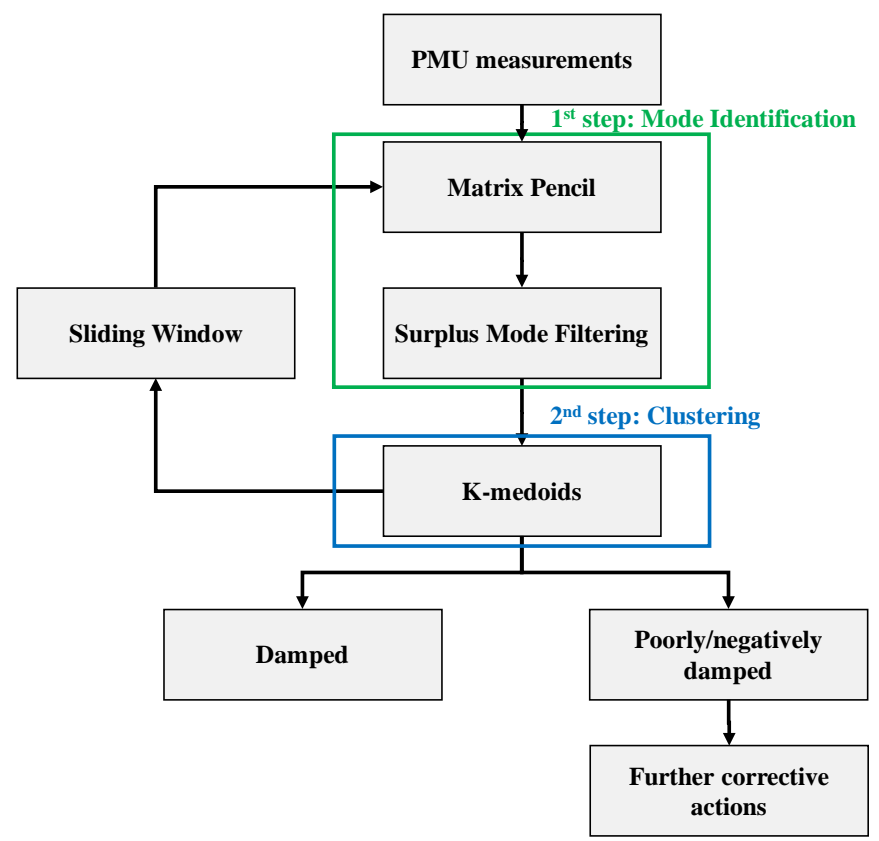

Fig. 1. Schematic of the application of the proposed method.

$$
\hat{y}[n]=\sum_{i=1}^{N} B_{i} z_{i}^{n}
$$

where:

$$
Z_{i}=e^{\lambda_{i} \Delta t}
$$

Thus, the signal is described by a finite summation of $p$ mode pairs $\left(B_{i}, z_{i}\right)$, where $B_{i}$ is the residue of the corresponding discrete-time pole $z_{i}$. The problem is to minimize the error between the real $y[n]$ and the estimated $\hat{y}[n]$ responses, by calculating accordingly the mode pairs of (2) as shown in (4).

$$
\min J=\sum_{n=0}^{N_{w}-1}(y[n]-\hat{y}[n])^{2}
$$

For this purpose, the MP method is adopted, which uses singular value decomposition (SVD) [16] to estimate the system modes, by filtering out noise or any additional components. The MP algorithm is summarized as follows [16]:

1) Build Hankel matrices $\mathbf{H}_{0}$ and $\mathbf{H}_{1}$ with entries the samples of the ringdown response $y[n]$ of the system.

2) Perform SVD of $\mathbf{H}_{0}$, defined in (5), and estimate the system order by retaining the largest singular values.

$$
\mathbf{H}_{0}=\mathbf{P S Q}^{T}
$$

3) Build vectors $\mathbf{V}_{1}$ and $\mathbf{V}_{2}$ by deleting the last and the first row of the unitary vector $\mathbf{V}_{\mathrm{N}}$, respectively, whose elements correspond to most significant values of $\mathbf{S}$. Calculate matrices $\mathbf{Y}_{1}$ and $\mathbf{Y}_{2}$ by:

$$
\begin{aligned}
& \mathbf{Y}_{1}=\mathbf{V}_{1}^{T} \mathbf{V}_{1} \\
& \mathbf{Y}_{2}=\mathbf{V}_{2}^{T} \mathbf{V}_{1}
\end{aligned}
$$

4) Poles $z_{i}$ are the generalized eigenvalues resulting from:

$$
\mathbf{Y}_{1}^{-1} \mathbf{Y}_{2}-\lambda \mathbf{I}=\mathbf{0}
$$

Since the system modes are identified by means of (3) using the calculated $z_{i}$, (8) can be solved in the least squares sense to estimate the associated residues $B_{i}$.

$$
\left[\begin{array}{cccc}
z_{1}^{0} & z_{2}^{0} & \mathrm{~L} & z_{N}^{0} \\
z_{1}^{1} & z_{2}^{1} & \mathrm{~L} & z_{N}^{1} \\
\mathrm{M} & \mathrm{M} & \mathrm{O} & \mathrm{M} \\
z_{1}^{N_{\mathrm{w}}-1} & z_{2}^{N_{\mathrm{w}}-1} & \mathrm{~L} & z_{N}^{N_{\mathrm{w}}}-1
\end{array}\right]\left[\begin{array}{c}
B_{1} \\
B_{2} \\
\mathrm{M} \\
B_{N}
\end{array}\right]=\left[\begin{array}{c}
y[0] \\
y[1] \\
\mathrm{M} \\
y\left[N_{w}-1\right]
\end{array}\right]
$$

To evaluate the accuracy of the identified modes the $R^{2}$ coefficient, defined in (9), is adopted, where $\bar{y}$ is the mean of $y[n]$. This $R^{2}$ examines the quality of the reconstructed signal to the original response fit.

$$
R^{2}=1-\frac{\sum_{n=1}^{N_{w}}(y[n]-\hat{y}[n])^{2}}{\sum_{n=1}^{N_{w}} y[n]-\bar{y}}
$$

\section{B. Surplus Mode Filtering Based on Energy}

The application of the MP to measured responses will result in the identification of the dominant modes contained in the ringdown as well as into a number of artificial surplus modes, due to noise, etc. These modes are typically characterized by low energy and might sometimes exhibit low or even negative damping [22]. Since the least damped mode of each generator is selected as an input parameter to the clustering algorithm, it is very important to exclude/separate the surplus modes from the dominant ones to reduce the number of possible false alarms. For this purpose, the normalized energy of each mode $\left(E_{i, n}\right)$ is calculated according to (10) and (11). Modes with less than $1 \%$ normalized energy (empirically chosen in this case) are considered artificial and discarded from the procedure.

$$
\begin{gathered}
E_{i}=\sum_{j=0}^{N_{\mathrm{w}}+1}\left(2 \operatorname{real}\left(B_{i} z_{i}^{j}\right)\right)^{2} \\
E_{i, n}=\frac{E_{i}}{\sum_{i=1}^{N} E_{i}}
\end{gathered}
$$

It should be noted that more advanced mode identification techniques automatically determining the required order could systematically solve this issue, thus additional filtering might not be essential. However, such methods could increase significantly the computational burden by introducing additional computations, e.g. by iterative loops [18].

\section{Unsupervised Machine Learning}

Since the dominant modes of the response of each generator are obtained following the proposed mode identification 
procedure, the least damped mode (including negatively damped modes) is selected as the input feature for the clustering algorithm. The clustering algorithm is applied for the least damped mode obtained for the initial time window $T_{w}$ as well as for any consecutive sliding windows as explained above.

K-medoids is used for two main reasons. First, the target of the proposed algorithm is to distinguish between generators exhibiting poorly/negatively damped and well damped oscillatory behavior. The number of clusters is therefore constant $(k=2)$, thus $\mathrm{k}$-means and $\mathrm{k}$-medoids with predefined number of clusters can be considered as a good choice. A larger number of clusters (e.g., 3, 4 etc.), however, could be defined by a system operator, as a part of the proposed methodology, if more detailed information on the generator groupings was desirable. Additionally, the k-medoids algorithm offers the advantage over k-means that the cluster centroid is not the mean value of the cluster objects but one of the actual objects of the cluster. This means that the cluster centroid corresponds to the actual least damped mode associated with one of the generators belonging to each cluster and therefore has a physical meaning.

The k-medoids algorithm is very similar to k-means and aims at clustering the objects of a dataset (generators in this case) into $k$ groups based on a number of features (the frequency and damping ratio of the least damped mode in the examined problem). Therefore, a number of $G$ observations corresponding to the total number of generators in the system occur, each characterized by two features. Therefore, a $2 \times G$ matrix with information obtained from the mode identification step is used as input to the clustering algorithm.

K-medoids is a partitioning technique that uses one representative object in each cluster as a reference point to the entire cluster. The Partitioning Around Medoids (PAM) algorithm is used in this paper, which is one of the most popular realizations of the k-medoids clustering [20]. The overall goal is to minimize an absolute error criterion presented in (12), where $e$ is the sum of absolute error for all objects $\mathbf{p}$ of the data set, $\mathbf{o}_{i}$ is the representative object of the cluster $C_{i}$ and dist is the selected distance measure which in this case is the Euclidean distance. Initially, a representative object for each cluster is chosen either arbitrarily or as in this paper following the k-means++ algorithm to speed up the process [23]. An iterative approach is followed in a greedy manner by changing the representative object of a cluster and calculating whether the absolute error is reduced or not. At each iteration the representative object is modified to the new one if the error is reduced, otherwise the same representative object is used.

$$
e=\sum_{i=1}^{k} \sum_{p \in C_{i}} \operatorname{dist}\left(\mathbf{p}, \mathbf{o}_{i}\right)
$$

\section{Sliding Window Method}

The initial selected signal time window $T_{w}$ is assumed long enough to contain most of the signal energy associated with the system oscillatory modes [18]. However, by applying the above procedure only to $T_{w}$, it may lead to the elimination of important low/negatively damped oscillatory modes, characterized by low energy during this period. To overcome this issue, the MP and surplus mode filtering procedures can be additionally applied to consecutive sliding signal windows of length $T_{w}$. As the energy of these modes increases with time, their identification can be eventually successful at a later time step and the updated "list" of poorly damped modes provided to the clustering algorithm.

\section{E. Probabilistic Oscillatory Behaviour}

In order to account for the increasing uncertainty in modern power system operation, a probabilistic approach is followed to investigate the probabilistic oscillatory behaviour of the system as well as of individual generators. The uncertainty of the intermittent behaviour of RES and system loading is taken into consideration to produce a number of TD dynamic MC simulations for different operating conditions. The number of simulations required is defined according to the required error of the sample mean shown in (13), where $\Phi^{-1}$ is the inverse Gaussian cumulative distribution function (CDF) with a mean value of zero and standard deviation one, $\sigma^{2}$ is the variance of the sampled random variable $X_{N}, \delta$ is the confidence level (i.e. 0.01 for this study) and $N_{M C}$ the number of MC samples [5].

$$
e_{\bar{X}_{N}}=\frac{\Phi^{-1}\left(1-\frac{\delta}{2}\right) \sqrt{\frac{\sigma^{2}\left(X_{N}\right)}{N_{M C}}}}{\bar{X}_{N}}
$$

\section{TEST SYSTEM, UNCERTAINTIES AND SiMULATIONS}

\section{A. System Under Study}

Dynamic simulations are used to substitute measurement data. The test network used is a modified version of the IEEE 68 bus, 16 machine reduced order equivalent model of the New England Test System and the New York Power System (NETS - NYPS). The conventional part of the test network is adopted from [9] and RES are added at the buses shown in Fig. 2. Two types of RES units are connected on each bus: Doubly Fed Induction Generators (DFIGs), representing wind generators (WGs) and Full Converter Connected (FCC) units, representing both WGs and photovoltaic (PV) units. The dynamic performance of the system is analyzed by means of RMS simulations using DIgSILENT - PowerFactory software [26].

Standard $6^{\text {th }}$ order models are used for all synchronous generators including either slow IEEE DC1A dc exciters or fast acting static exciters type IEEE ST1A and generic governors, representing gas, steam and hydro turbines. Generator G9 is also equipped with a Power System Stabilizer (PSS).

A generic type 3 model, suitable for large scale stability studies, is used in this paper to represent DFIGs and a type 4 WG model is used to represent all FCC units (both WGs and PVs). The models have a structure similar to the one proposed by WECC [24] and IEC [25], and are available in DIgSILENT - PowerFactory [26], whereas all RES units are 
considered with Fault Ride Through (FRT) capabilities.

The number of the connected RES corresponds to $20 \%$ of the total installed generating capacity of the system, $66.67 \%$ of which are type 3 DFIGs and 33.33\% FCC units. FCCs are further considered to be $30 \%$ WGs and $70 \%$ PV units.

\section{B. Modelling of Uncertainties}

The time frame for the considered uncertainties in this study is one day. Therefore, daily loading and PV curves are initially used and the hour of the day is sampled randomly following a uniform distribution to determine the pu values for all the loads and PV units. For every hour within the day, the corresponding uncertainties are also modeled using a normal distribution for the system load and a beta distribution for PV generation. Therefore, an extra uncertainty scaling factor for loads and PVs is introduced, which is eventually multiplied with the corresponding value from the daily loading or PV curve, respectively. The normal distribution for the system loading uncertainty has mean value 1 pu and standard deviation 3.33\% and the beta distribution $a$ and $b$ parameters are 13.7 and 1.3, respectively. The uncertainty of the wind speed is modelled using a Weibull distribution with parameters $\varphi=11.1$ and $k=2.2$ [13].

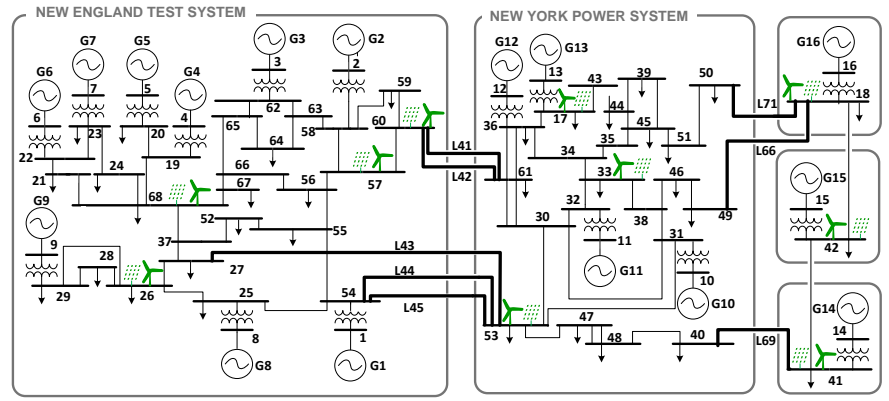

Fig. 2. Modified IEEE 68 bus test network.

\section{Simulation Procedure}

After considering the uncertainties, Optimal Power Flow (OPF) is solved to determine the conventional generators dispatch. The nominal capacity of each generator is then adjusted to take into consideration the disconnection of conventional generation due to both load variations and RES penetration. A constant amount of $15 \%$ spare capacity is considered for each simulated case as in [27]. Since the generators are considered as aggregated units, reducing the nominal power is equivalent to a reduction in the moment of inertia of the power plant and an increase in the generator reactance.

Three phase self-clearing faults are considered as disturbances in this study. However, the simulation database could be extended to include any other disturbances as well. A uniform distribution is used to model the fault location which means that the fault may happen with equal probability at any line of the test network and at any point along the line. A normal distribution with mean value of 14 cycles and standard deviation $6.67 \%$ is used to model the fault duration.

TD dynamic RMS simulations are performed afterwards for the operating conditions resulting from OPF and the selected contingencies explained above, in DIgSILENT PowerFactory [26]. For the examined system the number of required MC simulations $\left(N_{M C}\right)$ is 6000 to limit the error of the sample mean described in (13) to 5\% [5].

\section{CASE STUDIES}

The selected time window (after the fault is cleared) in studies carried out to illustrate the proposed methodology is approximately 18 seconds, i.e. 1077 samples with a sampling time of 1 cycle (in $60 \mathrm{~Hz}$ ). The order for the adopted MP method is 10 , resulting in the identification of 5 oscillatory modes. This model order is selected to ensure that the median of the $R^{2}$ values for all generators and for all simulated cases is higher than $95 \%$. The frequencies of interest in this study are those $>0.1 \mathrm{~Hz}$ to focus on electromechanical modes.

\section{A. Application to Representative Cases}

Two representative Test Cases (TCs) of generator oscillatory behaviour obtained from MC simulations are shown in Fig. 3. In the first case, i.e. TC1, all generators exhibit well damped oscillatory behaviour, while in TC2 some generators exhibit negatively damped oscillations with slowly growing amplitudes. The least damped modes of each generator are initially identified and afterwards used as inputs for the k-medoids algorithm.

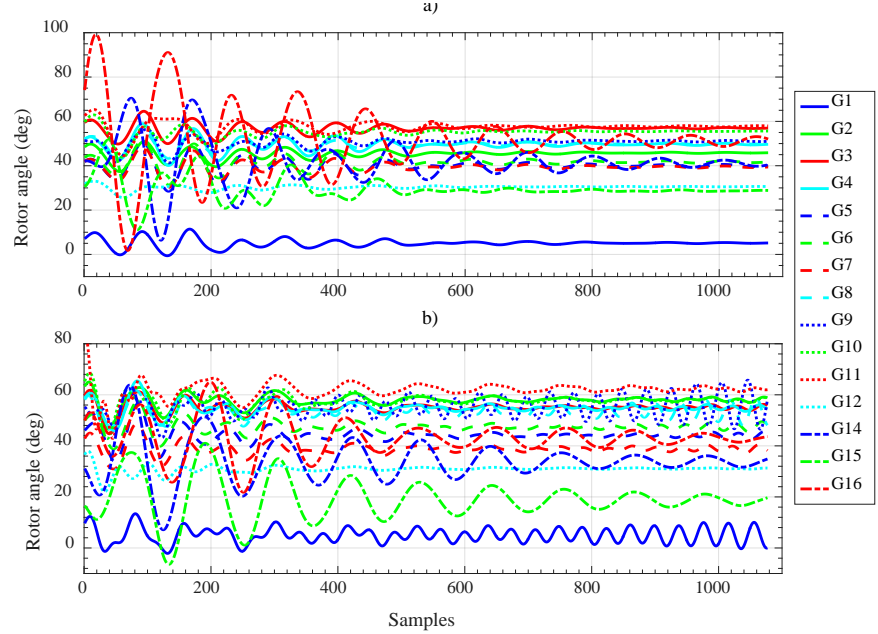

Fig. 3. Representative responses for a) well damped (TC1) and b) negatively damped (TC2) response.

The identified least damped modes for TC1 are presented in Fig. 4a. The clusters obtained by applying the k-medoids algorithm are also marked in Fig. 4a along with the representative object of each cluster. For TC1, the least damped modes for all generators have positive damping ratio and therefore both resulting groups exhibit stable oscillatory behaviour. Each group is related to a representative generator (defined as the representative object of the cluster following the k-medoids clustering algorithm), which describes the oscillatory behaviour of the generators that belong to each group, i.e. G15 (damping ratio $3.87 \%$ and frequency $0.86 \mathrm{~Hz}$, representing the group of G8) and G1 (damping ratio 4.3\% and frequency $0.57 \mathrm{~Hz}$, representing the group of G2-G7 and G9-G14) in this case, respectively. The physical meaning of 
this grouping is that the generators can be split in two groups based on the frequency and damping ratio of their least damped mode due to more pronounced separation in frequency of the critical modes. Since in TC1 all generators exhibit stable oscillatory behaviour no further control action would be required. In TC2 however, one of the groups, namely, the critical group, exhibits growing oscillations. This critical group is represented by generator G6 (damping ratio $1.6 \%$ and frequency $1.57 \mathrm{~Hz}$ ) and includes G1, G4, G7, G8 and G9. The rest of the generators exhibit stable oscillatory responses within this time window and belong to the group represented by G10 (corresponding to damping ratio 4.27\% and frequency $0.53 \mathrm{~Hz}$ ).

\section{B. Filtering of Artificial Surplus Mode}

Fig. 5, shows an example of a representative response of G1, where artificial low energy modes appear (as explained in Section II.B), whereas Table I summarizes the extracted modes from the application of the MP method, without considering surplus filtering. In Fig. 5a, the original and reconstructed signals using the MP method are compared. In Fig. 5b, the damped sinusoidal responses of each of the 5 identified modes are analyzed individually. Although the fit between the two signals ( $R^{2}$ value of $98.4 \%$ ) is very good, two of the extracted modes, i.e. mode \#1 and \#5, are characterized by very low energy (as shown in the zoomed version of the plot) as well as by very low or negative damping as described in Section II.B. The normalized energy of mode \#1 and mode \#5 is $1.9 \mathrm{e}^{-2}$ and $1.39 \mathrm{e}^{-5}$, respectively and thus they will be filtered out when calculating the least damped mode, following the proposed procedure. If these trivial modes characterized by very low energy (as can also be seen in Fig. 5b) were not filtered out, the least damped mode would end up being mode \#1. Therefore, the proposed surplus mode filtering procedure acts as an additional measure to ensure that only the important modes are included in the second step of the algorithm, while the trivial ones are filtered out.
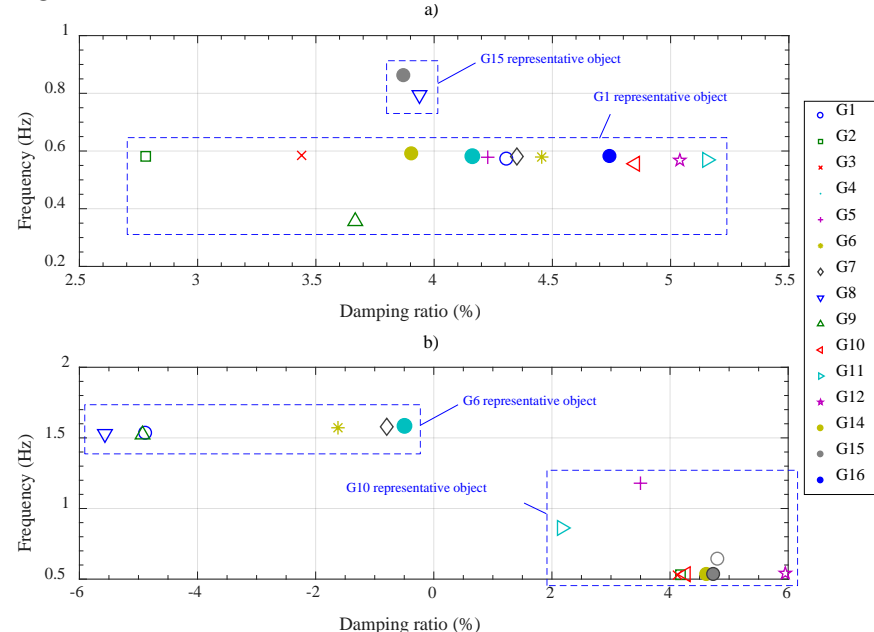

Fig. 4. Generator groups obtained from k-medoids clustering for a) TC1 and b) TC2.

TABLE I

G1 EXTRACTED MODES (WITHOUT SURPLUS MODE FILTERING) \begin{tabular}{|l|l|l|l|} 
Mode & Frequency (Hz) & Damping Ratio (\%) & Normalized Energy \\
\hline
\end{tabular}

\begin{tabular}{|l|c|c|c|}
\hline $\mathbf{1}$ & 1.53 & $-1.74 \mathrm{e}-3$ & $1.9 \mathrm{e}-3$ \\
\hline $\mathbf{2}$ & 1.37 & 2.07 & $3.69 \mathrm{e}-2$ \\
\hline $\mathbf{3}$ & 0.78 & 4.7 & 0.895 \\
\hline $\mathbf{4}$ & 0.51 & 11.42 & $6.6 \mathrm{e}-2$ \\
\hline $\mathbf{5}$ & 0.27 & 2.03 & $1.39 \mathrm{e}-5$ \\
\hline
\end{tabular}

a)
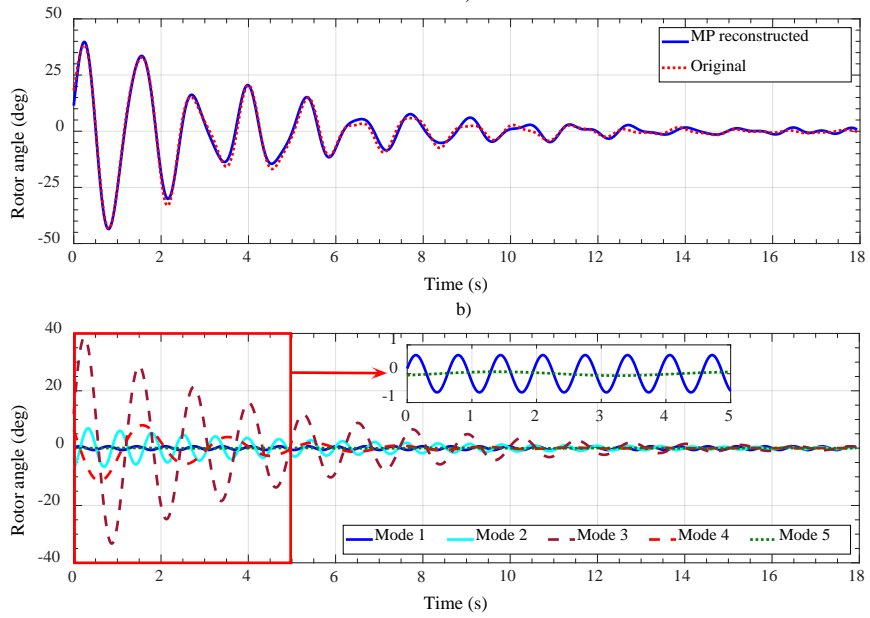

Fig. 5. G1 response and obtained modes from MP method.

\section{Application of Sliding Window}

In some cases, certain generators might start exhibiting oscillatory behaviour after the selected time window used in the system identification procedure. For this purpose (as explained in Section II.C), a sliding window is applied. To highlight the importance of the sliding window technique the following case is investigated. Let us assume that the window is of fixed length and moves by 1 sample as each new measurement point is received. To illustrate this, the response of G2 for TC2 presented before is shown in Fig. 6a for an overall duration of 1107 samples (100 additional samples compared to Fig. 3). The sliding window, denoted with the red box, has a duration of 1077 samples and as time proceeds moves to the right 100 times for this specific case shown in Fig. 6. The method is therefore applied 100 times as the window slides. As time goes by, generator G2 is exhibiting growing oscillations which were not included in the initial time window (and therefore have not been identified by the MP method).

Fig. 6b presents the least damped mode identified by applying consecutively the mode identification method as the window slides. As mentioned above the method is applied 100 times as each new sample is obtained from the measurements. These generated 100 points in Fig. $6 \mathrm{~b}$ start from the left for the initial window and move to the right, as the window slides. It can be seen that only after 46 samples (also denoted in Fig. $6 b)$, the unstable mode corresponding to the growing oscillations is successfully identified, jumping from a positive damping value close to $4 \%$ to a negative damping value close to $-1.9 \%$. It should be noted that the least damped mode identified using the initial window is also of different frequency, i.e. approximately $0.5 \mathrm{~Hz}$, while the unstable mode is close to $1.5 \mathrm{~Hz}$, which is the same frequency with the group of unstable generators already identified before. Therefore, as 
the window is moving, generator G2 will eventually move to the unstable cluster.
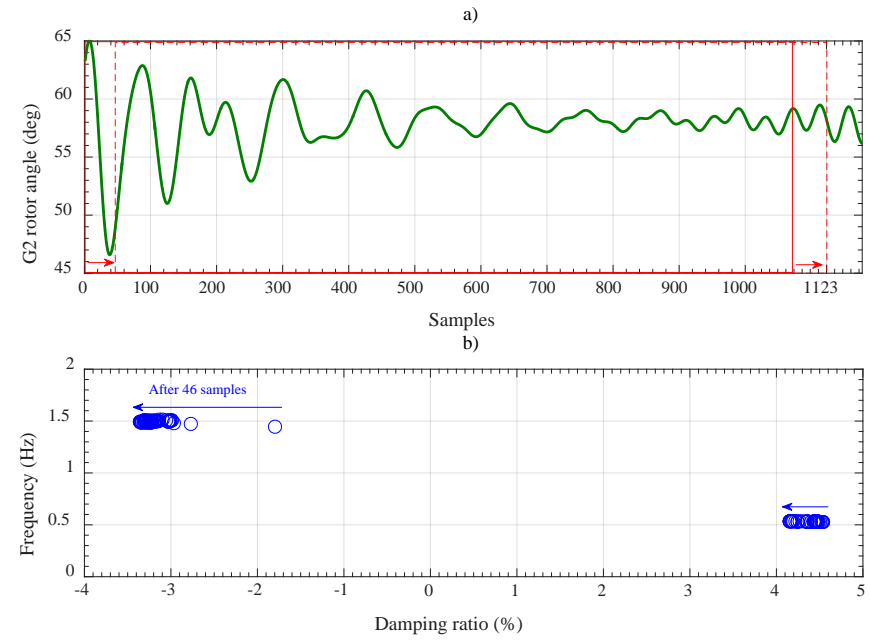

Fig. 6. a) G2 response and sliding window and b) least damped mode as window slides.

\section{ANALysis of Probabilistic OsCILlatory Behaviour OF THE SYSTEM AND INDIVIDUAL GENERATORS}

The application of the proposed methodology to the results of MC simulations carried out to account for the uncertain power system behavior is illustrated below. A boxplot of the $R^{2}$ values for all the simulated cases and all generators is shown in Fig. 7 to highlight the performance of the mode identification method. The median of the $R^{2}$ is $>95 \%$ for all generators, ranging from $99.9 \%$ for G5 and $93.7 \%$ for G8. The $R^{2}$ in most cases remains $>80 \%$ though in few cases the outliers with low $R^{2}$ values appear, especially for generators G1 and G8. The $R^{2}$ though is $<50 \%$ only in $0.04 \%$ for G16 and $0.9 \%$ for G8 of the total number of simulated cases. This is mainly caused by the fixed identification model order of the MP method used during the mode identification step. In some cases though, a higher identification model order might be necessary to achieve higher $R^{2}$ value.

\section{A. Probabilistic Assessment of Generator Groups}

The proposed methodology is also applied to all first-swing stable cases, i.e. 5345 out of 6000 performed simulations. The resulting representative objects of the two clusters for each case are summarized in Fig. 8 (focusing on the range with damping between $\pm 10 \%$ ). Blue circles correspond to the representative object of the group with lower damping, while the green squares are the representative objects corresponding to the well damped group. It should be noted that there are some cases where both groups might exhibit stable or unstable oscillatory behaviour. Observing the location of the representative objects (i.e. the damping ratio and frequency) is therefore important to draw conclusions regarding the oscillatory behaviour of the generators belonging to each cluster. Additionally, it helps in identifying the modes that might cause unstable behaviour of the system under uncertainties considered (intermittent nature of wind generation, PVs and system loading in this particular case). It can be seen, for example, that some modes located in the region around $0.5 \mathrm{~Hz}$ can exhibit low (but positive) damping and only in very few cases might become unstable. On the other hand, some modes in the frequency region close to $1.1 \mathrm{~Hz}$ tend to exhibit unstable behaviour in several cases, followed by modes with frequencies ranging from $1.2 \mathrm{~Hz}$ to $1.6 \mathrm{~Hz}$.

In order to get a more detailed overview of the dynamic behaviour of the system, some representative generator grouping patterns (possible cluster formations within the simulated cases) are presented in Table II, along with the number of occurrences of each pattern. The total number of the observed grouping patterns is 796 for the 5345 first-swing stable cases of the examined dataset with a large number of them occurring less than 10 times. However, as noted before, the exact location of the representative cluster object (representative generator) should be observed to get a more accurate description of the cluster (whether it exhibits well or poorly damped oscillations). This means, for example, that in some of the grouping patterns presented in Table II, both groups might be well damped (with one group being less damped than the other). In this way, the information on the expected grouping patterns as well as on the frequency of their appearance for a given system is provided.

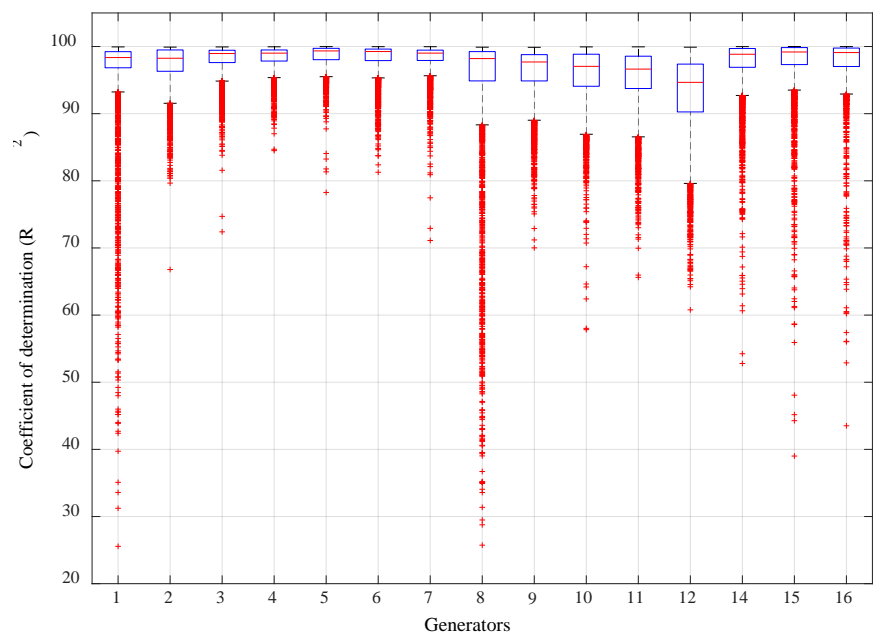

Fig. 7. $R^{2}$ for all cases for each generator.

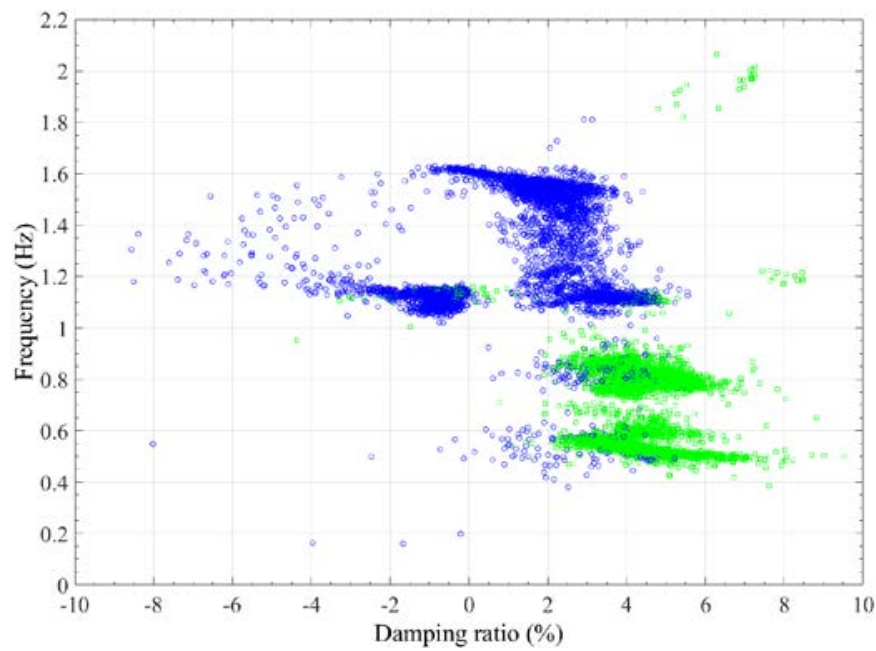

Fig. 8. Representative cluster objects. 
In Fig. 9, the representative objects of the two clusters for pattern \#5 of Table II are presented in a similar manner to Fig. 8. For this specific pattern, the entire NETS area and one generator in NYPS area (G1-G10) exhibit unstable oscillatory behavior with frequency around $1.1 \mathrm{~Hz}$ while the rest of the generators (G11-G16) oscillate with positively damped oscillations with frequencies that vary from $0.5 \mathrm{~Hz}$ to $0.9 \mathrm{~Hz}$.

\section{B. Probabilistic Dynamic Behaviour of Individual Generators}

By observing the extracted least damped mode for a specific generator for all cases, the individual generator oscillatory behaviour can be investigated. Results for G4 are presented in Fig. 10a. For this specific generator, the mode around $1.1 \mathrm{~Hz}$ might cause unstable or poorly damped (damping ratio less than 2\%) oscillatory behaviour. Additionally, it is less likely for two modes with frequency $1.4 \mathrm{~Hz}$ and $1.6 \mathrm{~Hz}$ to exhibit unstable oscillations. Similarly, results for G15 are presented in Fig. 10b revealing that this generator exhibits unstable modes very rarely, and if so, mostly with a frequency around $1.1 \mathrm{~Hz}$. Most of the times, G15 oscillates with frequencies around $0.5 \mathrm{~Hz}$ and $0.8 \mathrm{~Hz}$. Of the two, the $0.8 \mathrm{~Hz}$ mode is less damped and occasionally, though very rarely, might exhibit poor damping (less than $1 \%$ damping ratio).

TABLE II

REPRESENTATIVE GROUPING PATTERNS

\begin{tabular}{|c|c|c|c|}
\hline \multirow{2}{*}{$\begin{array}{c}\text { Pattern } \\
\text { No. }\end{array}$} & \multicolumn{2}{|c|}{ Grouping Pattern } & $\begin{array}{c}\text { Number of } \\
\text { occurrences }\end{array}$ \\
\cline { 2 - 3 } & $\begin{array}{c}\text { Less damped } \\
\text { group }\end{array}$ & $\begin{array}{c}\text { Better damped } \\
\text { group }\end{array}$ & \\
\hline $\mathbf{1}$ & $(\mathrm{G} 1-\mathrm{G} 12)$ & (G14-G16) & 1311 \\
\hline $\mathbf{2}$ & $(\mathrm{G} 1, \mathrm{G} 8, \mathrm{G} 9)$ & $(\mathrm{G} 2-\mathrm{G} 7, \mathrm{G} 10-\mathrm{G} 16)$ & 449 \\
\hline $\mathbf{3}$ & $\begin{array}{c}\text { (G1, G8, G9, } \\
\text { G12) }\end{array}$ & $\begin{array}{c}\text { (G2-G7, G10, G11, } \\
\text { G14-G16) }\end{array}$ & 276 \\
\hline $\mathbf{4}$ & $(\mathrm{G} 9)$ & (G1-G8, G10-G16) & 131 \\
\hline $\mathbf{5}$ & $(\mathrm{G} 1-\mathrm{G} 10)$ & (G11-G16) & 70 \\
\hline
\end{tabular}

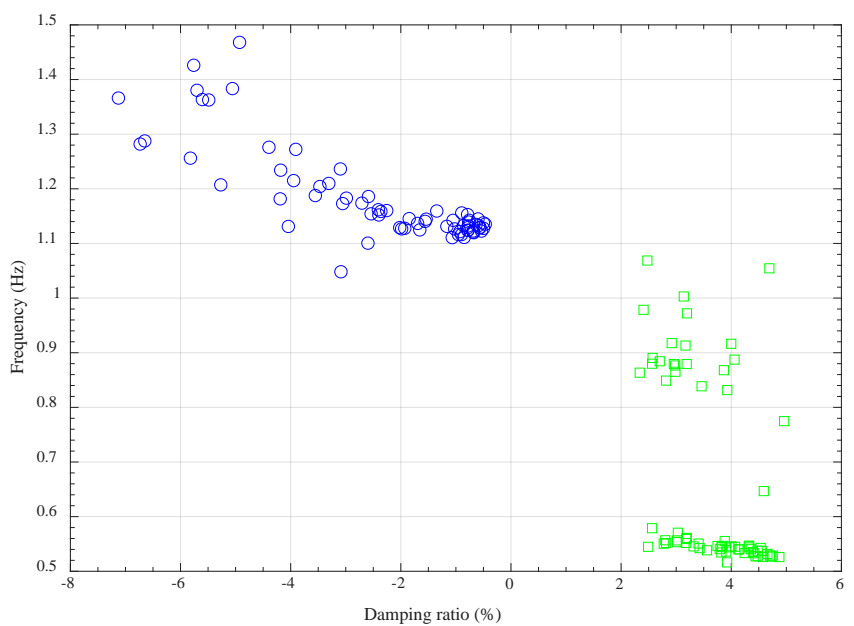

Fig. 9. Representative objects corresponding to a specific grouping pattern.

\section{Effect of RES on Individual Generator Oscillatory Behaviour}

An additional case study is performed, considering specifically the effect of RES units on individual generator oscillatory behaviour. In this case, the system loading is taken into account as the only uncertainty considering the operating conditions of the network (fault location and duration uncertainties remain as described before), since all RES units are disconnected. In Fig. 11, the Cumulative Distribution Functions (CDF) of the damping ratio of the least damped mode for three representative generators (G1, G4 and G15) are compared for the cases with and without RES. In general, there is higher probability that these three generators will exhibit negatively damped oscillations after the disturbance. More specifically, G4 for which the deterioration is more evident, has approximately $34 \%$ probability to exhibit negatively damped oscillations when RES are connected while the same probability is approximately $28 \%$ without RES. This denotes a possible increase in the probability of oscillatory instability following connection of RES of the specific generator (and the system in general) for a set of considered operating conditions.

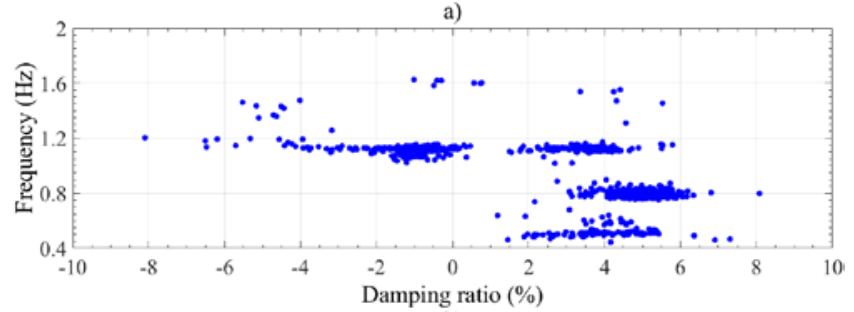

b)

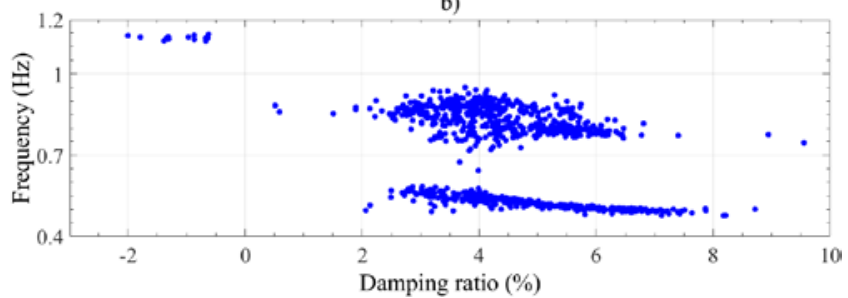

Fig. 10. Least damped identified modes for a) G4 and b) G15.

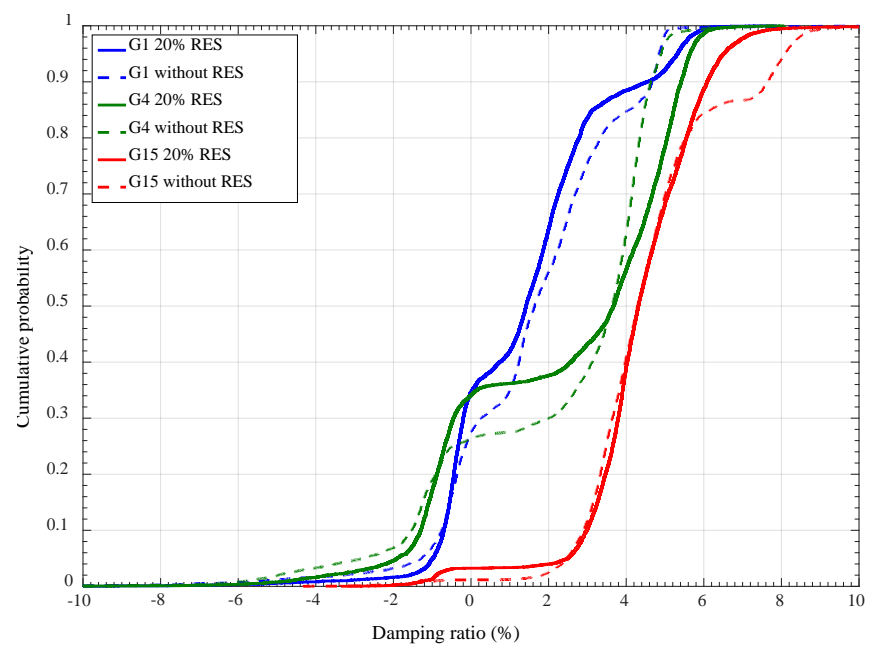

Fig. 11. CDFs of the damping ratio of the least damped mode for G1, G4 and G15 with and without RES.

\section{Computational Burden Considerations}

All computations have been performed using an Intel Core i7 $3.4 \mathrm{GHz}$ with $16 \mathrm{~GB}$ of RAM. Both MP mode identification and k-medoids clustering algorithms are implemented in Matlab. For the $1^{\text {st }}$ step (i.e. mode identification) of the 
proposed algorithm approximately 550ms in total are required for the extraction of the modes for all generators, after the measurements for the chosen time window are obtained. The $2^{\text {nd }}$ step (i.e. the clustering algorithm) requires approximately $25 \mathrm{~ms}$. Therefore, for a single time window calculation of the generator clusters, less than $600 \mathrm{~ms}$ are required, showing that the method is suitable for close to real-time applications.

\section{CONCLUSIONS}

In this paper, a two-step, measurement based method is proposed for the online identification and analysis of power system oscillatory behaviour. The proposed method is applied in a close to real-time manner (less than $600 \mathrm{~ms}$ ), to provide additional actionable information to system operators regarding the participation of individual generators in poorly or negatively damped oscillatory modes. First, the MP method is applied to TD responses to extract the dominant modes contained in both stable and unstable oscillatory responses of each generator in the system. Next, the least damped mode of each generator is used to cluster the generators in groups based on their oscillatory behaviour, using the k-medoids algorithm. A sliding window is used to capture possible growing oscillations that could have been missed using the initial fixed time window as well as a surplus mode filtering approach to ensure consistency and automate the procedure when applying the method on a large number of responses for diverse operating conditions (motivated by the intermittent behaviour of RES).

From the analysis performed, it can be concluded that the MP method can successfully and with high confidence identify both, stable and unstable, oscillatory modes of each generator. Moreover, the clustering analysis showed that generators can be successfully split into two distinct groups, each represented by a generator highlighting the oscillatory behaviour of the group.

Additionally, the application of the method has been demonstrated for the analysis of system dynamic behavior considering various uncertainties including the intermittent behaviour of RES. By applying the method on a large number of MC simulations, the oscillatory modes and generators that may cause instability of a system can be identified. Comparative study with and without RES showed that, for the specific system under study and for the operating conditions investigated, the probability of the appearance of negatively damped oscillations for certain generators might increase when RES are connected.

\section{REFERENCES}

[1] S. Rovnyak, S. Kretsinger, J. Thorp, and D. Brown, "Decision trees for real-time transient stability prediction," IEEE Trans. Power Syst., vol. 9, no. 3, pp. 1417-1426, 1994.

[2] V. Vittal, N. Senroy, and G. T. Heydt, "Decision Tree Assisted Controlled Islanding," IEEE Trans. Power Syst., vol. 21, no. 4, pp. 1790-1797, 2006.

[3] Q. Gao and S. M. Rovnyak, "Decision trees using synchronized phasor measurements for wide-area response-based control," IEEE Trans. Power Syst., vol. 26, no. 2, pp. 855-861, 2011.
[4] M. He, J. Zhang, and V. Vittal, "Robust Online Dynamic Security Assessment Using Adaptive Ensemble Decision-Tree Learning,” IEEE Trans. Power Syst., vol. 28, no. 4, pp. 4089-4098, 2013.

[5] P. N. Papadopoulos, T. Guo, J. V. Milanovic, "Probabilistic Framework for Online Identification of Dynamic Behavior of Power Systems with Renewable Generation,” IEEE Trans. Power Syst., available online.

[6] F. R. Gomez, A. D. Rajapakse, U. D. Annakkage, and I. T. Fernando, "Support vector machine-based algorithm for post-fault transient stability status prediction using synchronized measurements," IEEE Trans. Power Syst., vol. 26, no. 3, pp. 1474-1483, 2011.

[7] N. Amjady, S. F. Majedi, "Transient stability prediction by a hybrid intelligent system," IEEE Trans. Power Syst., vol. 22, no. 3, pp. 12751283, 2007.

[8] S. Kretsinger, S. Rovnyak, D. Brown, and J. Thorp, "Parallel decision trees for predicing groups of unstable generators from synchronized phasor measurements," in Proc. Precise Measurements in Power Systems Conf., Washington, DC, USA, 1993.

[9] G. Rogers, Power System Oscillations. Kluwer Academic, 2000.

[10] M. Jonsson, M. Begovic, J. Daalder, "A new method suitable for realtime generator coherency determination," IEEE Trans. Power Syst., vol. 19, no. 3, pp. 1473-1482, 2004.

[11] M. A. M. Ariff, B. C. Pal, "Coherency Identification in Interconnected Power System-An Independent Component Analysis Approach,” IEEE Trans. Power Syst., vol. 28, no. 2, pp. 1747-1755, 2013.

[12] P. McNabb, D. Wilson, J. Bialek, "Classification of mode damping and amplitude in power systems using synchrophasor measurements and classification trees," IEEE Trans. Power Syst., vol. 28, no. 2, pp. 19881996, 2013.

[13] P. N. Papadopoulos, J. V. Milanovic, P. Bhui, N. Senroy, "Feasibility Study of Applicability of Recurrence Quantification Analysis for Clustering of Power System Dynamic Responses”, in ISGT Europe 2016, Ljubljana, Slovenia, November, 2016.

[14] J. F. Hauer, C. J. Demeure, L. L. Sharf, "Initial results in Prony analysis of power system response signals," IEEE Trans. Power Syst., vol. 5, no. 1, pp. 80-89, 1990.

[15] J. J. Sanchez-Gasca, "Computation of turbine-generator subsynchronous torsional modes from measured data using the eigensystem realization algorithm,"'IEEE Power Eng. Soc. Winter Meet., Columbus, USA, 2001.

[16] M. L. Crow, A. Singh, "The matrix pencil for power system modal extraction,” IEEE Trans. Power Syst., vol. 20, no. 1, pp. 501-502, 2005.

[17] Z. Tashman, H. Khalilinia, V. Venkatasubramanian, "Multi-dimensional Fourier ringdown analysis for power systems using synchrophasors," IEEE Trans. Power Syst., vol. 29, no. 2, pp. 731-741, 2014.

[18] T. A. Papadopoulos, A. I. Chrysochos, E. O. Kontis, P. N. Papadopoulos, G. K. Papagiannis, "Measurement-Based Hybrid Approach for Ringdown Analysis of Power Systems," IEEE Trans. Power Syst., vol. 31, no. 6, pp. 4435-4446, 2016.

[19] "IEEE Standard for Synchrophasor Measurements for Power Systems," IEEE Std C37.118.1-2011 pp. 1-61.

[20] J. Han, M. Kamber, J. Pei, Data mining concepts and techniques. Waltham, USA: Elsevier, 2012.

[21] IEEE Task Force on Identification of Electromechanical modes, "Identification of electromechanical modes in power systems," IEEE Power \& Energy Society, Tech. Rep. PES-TR15, 2012. [Online]. Available: http://resourcecenter.ieee-pes.org/.

[22] N. Zhou, J. W. Pierre, D. Trudnowski, "A Stepwise Regression Method for Estimating Dominant Electromechanical Modes," IEEE Trans. Power Syst., vol. 27, no. 2, pp. 1051-1059, 2012.

[23] Arthur, David, and Sergi Vassilvitskii. "K-means++: The Advantages of Careful Seeding”, in SODA '07: Proceedings of the Eighteenth Annual ACM-SIAM Symposium on Discrete Algorithms, pp. 1027-1035, 2007.

[24] WECC Wind Power Plant Dynamic Modeling Guide, WECC Renewable Energy Modeling Task Force, 2014.

[25] Wind turbines - Part 27-1: Electrical simulation models - Wind turbines, IEC 61400-27-1, 2015.

[26] DIgSILENT-PowerFactory User Manual, DIgSILENT GmbH, 2014.

[27] P. N. Papadopoulos, J. V. Milanović, "Probabilistic Framework for Transient Stability Assessment of Power Systems With High Penetration of Renewable Generation,” IEEE Trans. Power Syst., vol. 32, no. 4, pp. 3078-3088,

Panagiotis N. Papadopoulos (S'05-M'14) received the Dipl. Eng. and Ph.D. degrees from the Department of Electrical and Computer Engineering at the Aristotle University of Thessaloniki, in 2007 and 2014, respectively. Since 2014 he has been postdoctoral Research Associate at the University of 
Manchester. He is currently a Lecturer in the Department of Electronic and Electrical Engineering at the University of Strathclyde. His special interests are in the field of power system modeling, simulation and investigation of dynamic behaviour of power systems with increased penetration of non-synchronous generation.

Theofilos A. Papadopoulos (S'01-M'09-SM'18) received the Dipl. Eng. and Ph.D. degrees from the School of Electrical and Computer Engineering at the Aristotle University of Thessaloniki, Greece, in 2003 and 2008, respectively. He is currently Asst. Professor at the Power Systems Laboratory of the Department of Electrical and Computer Engineering of the Democritus University of Thrace, Greece. His special interests are power systems modeling, PLC and computation of electromagnetic transients. He received the Basil Papadias Award at the IEEE PowerTech 07 Conference.

Andreas I. Chrysochos (S’08-M’16) received the Dipl. Eng. and Ph.D. degrees from the School of Electrical and Computer Engineering at the
Aristotle University of Thessaloniki, Greece, in 2009 and 2015 respectively. From 2015 to 2017, he was a Research Fellow at the same school. Since 2017, he has been a Senior Engineer at the R\&D Department of Cablel ${ }^{\circledR}$ Hellenic Cables S.A., Viohalco Group. His special interests are power systems modeling, computation of electromagnetic transients, and PLC. He was a scholar of the Alexander S. Onassis Public Benefit Foundation (2012-2015, 2016-2017).

Jovica V. Milanović (M'95, SM'98, F'10) is a Professor of Electrical Power Engineering, Deputy Head of School and Director of External Affairs in the School of Electrical and Electronic Engineering at the University of Manchester, U.K. Professor Milanovic is a Chartered Engineer in the UK, Foreign member of the Serbian Academy of Engineering Sciences, Fellow of the IET, Fellow of the IEEE, Distinguished IEEE PES Lecturer and currently serves on IEEE PES Governing Board as Regional Representative for Europe, Middle East and Africa and on IEEE PES Fellows Committee. 